\title{
MORSE THEORY OF HARMONIC FORMS
}

\author{
Michael Farber, Gabriel Katz and Jerome Levine
}

\begin{abstract}
We consider the problem of whether it is possible to improve the Novikov inequalities for closed 1-forms, or any other inequalities of a similar nature, if we assume, additionally, that the given 1-form is harmonic with respect to some Riemannian metric. We show that, under suitable assumptions, it is impossible. We use a theorem of E.Calabi [C], characterizing 1-forms which are harmonic with respect to some metric, in an essential way. We also study some interesting examples illustrating our results.
\end{abstract}

\section{StATEMENT OF RESUlTS}

1. Introduction. The Morse theory of closed 1-forms was begun by S. P. Novikov $[\mathrm{N}],[\mathrm{N} 1]$; a survey can be found in $[\mathrm{P}]$. The standard Morse inequalities for functions were generalized, by Novikov, to forms using a twisted cohomology defined by the 1-form in place of the usual cohomology. In some cases it is known that these inequalities are sharp $[\mathrm{F}],[\mathrm{P}]$.

In this paper we will address the problem of whether it is possible to improve these Novikov inequalities (or any other inequalities of a similar nature) assuming additionally that the given closed 1-form is harmonic with respect to some Riemannian metric. This problem was first considered in the paper of E.Calabi [C] even before Novikov's theory. There is one obvious restriction - namely, any nonconstant harmonic form has no local minima and maxima - and Calabi asked in [C] if there are any other restrictions on the critical point structure of Morse harmonic 1-forms.

Motivated by this, Calabi solved instead in [C] another fundamental problem: he gave a complete topological criterion for a closed Morse 1-form to be harmonic with respect to some Riemannian metric. We will call such forms intrinsically harmonic or Calabi forms. We will describe the theorem of Calabi briefly in Section 3.

The thrust of the main results in this paper is that, at least under certain quite general conditions, there exist no restrictions on the Morse numbers of harmonic forms except for the trivial restrictions mentioned above.

The conditions we need to impose are closely tied to the topological nature of the (singular) foliation which is associated to a Morse 1-form (see below for the definition). In particular, compactness of the leaves is a crucial item. Our results will show that if the foliation defined by a form $\omega$ is made up entirely of noncompact leaves or, at the other extreme, belongs to a cohomology class which is a

The research was supported by US - Israel Binational Science Foundation Grants 9400299 and 9400073, and by NSF Grant 93-03489.

Typeset by $\mathcal{A} \mathcal{M S}-\mathrm{T}_{\mathrm{E}} \mathrm{X}$ 
scalar multiple of an integral class (in which case all the leaves of the foliation are compact), then there is a Calabi form $\tilde{\omega}$ which is contiguous to $\omega$, i.e. it is in the same cohomology class and has the same number of critical points of each index.

We will also present some results concerning two issues related to the existence of compact leaves in the foliation of a closed Morse 1- form. In section 9 we show that such a form defines a natural decomposition of the manifold into pieces, each of whose interiors consists of all compact or all non-compact leaves. The combinatorics of this decomposition plays a crucial role in determining whether the form is contiguous to a Calabi form.

In Sections 10-11 we present two results which give restrictions on the cohomology class of a form when either all or none of the leaves of its foliation are compact. In Section 12 we describe several examples for surfaces which will illuminate and illustrate our results.

It is a theorem of Lalonde-Polterovich [LP] that, given any non-trivial class in $H^{1}(M, \mathbb{R})$, then, for a generic Riemannian metric, the harmonic form representing that class has only Morse singularities. In other words, generically harmonic forms have only Morse type singularities. This allows us to avoid considering more complicated singularities and leaves with the question addressed here of how many critical points these Morse harmonic forms can have.

Finally we would like to thank Robert Kotiuga for bringing the work of Calabi to our attention and for many stimulating discussions.

2. The main results. Here we formulate in a precise form our principal results.

Theorem 1. Suppose that $\omega$ is a closed Morse 1-form on a closed manifold $M$ which represents a scalar multiple of an integral cohomology class and such that $\omega$ has no critical points of index 0 or $n=\operatorname{dim} M$. Then there exists an intrinsically harmonic Morse 1-form $\tilde{\omega}$ which has the same numbers of critical points of all indices as $\omega$ and which is in the same cohomology class as $\omega$.

This theorem implies that, if there would exist Morse type inequalities for harmonic forms estimating the numbers of critical points by information depending only on the cohomology class of the form, they have to be also true for any closed 1-form representing a scalar multiple of an integral cohomology class. We refer to such cohomology classes as being of rank one. Following S.Novikov [N], we say that a cohomology class $\xi \in H^{1}(M, \mathbb{R})$ is of rank $k$ if the image of the homomorphism $\pi_{1}(M) \rightarrow \mathbb{R}$ determined by $\xi$ is a free abelian group of rank $k$.

We recall from $[\mathrm{N}]$ that, for a closed 1-form $\omega$ of rank one on a closed manifold $M$, the Novikov inequalities take the form:

$$
m_{p}(\omega) \geq b_{p}([\omega])+q_{p}([\omega])+q_{p-1}([\omega]),
$$

where $m_{p}(\omega)$ is the number of critical points of index $p$ and $b_{p}([\omega]), q_{p}([\omega])$ are the rank and torsion Novikov numbers of the cohomology of $M$ with local coefficients defined by $[\omega]$ (see $[\mathrm{N}]$ and $[\mathrm{F}]$ for precise definitions). In $[\mathrm{F}]$ it is shown that these inequalities are exact. Combining this with Theorem 1 gives:

Corollary. If $\pi_{1}(M)=\mathbb{Z}$ and $n=\operatorname{dim} M \geq 6$, then for any nonzero class $\xi \in$ $H^{1}(M ; \mathbb{R})$, there exists an intrinsically harmonic Morse form $\omega$ representing $\xi$ such that $m_{p}(\omega)=b_{p}(\xi)+q_{p}(\xi)+q_{p-1}(\xi)$. 
This follows by combining the main Theorem of $[\mathrm{F}]$ and Theorem 1 above and observing that, since $b_{0}(\xi)=q_{0}(\xi)=b_{n}(\xi)=q_{n-1}(\xi)=0$, the 1-form given by [F] realizing the given non-zero cohomology class $\xi$ has no critical points of indices 0 and $n$.

It is an easy fact that rank one Morse forms must have all compact leaves. In the next theorem we consider the opposite situation: we assume that all leaves are non-compact. In addition we need to assume that $\omega$ is generic, which we define to mean that any singular leaf contains only one critical point. It is easy to see that any Morse form can be perturbed slightly so that it becomes generic without changing the numbers of critical points or the cohomology class.

Theorem 2. If $\omega$ is a generic closed Morse 1-form all of whose leaves are noncompact then $\omega$ is intrinsically harmonic.

Theorem 2 is false if $\omega$ is not generic. See example (4) in Section 12.

Theorems 1 and 2 will be proved in Sections 7 and 8 .

\section{CAlabi's theOREM}

3. In this section we will recall Calabi's Theorem [C].

Let $\omega$ be a fixed closed 1-form on a closed manifold $M$.

A smooth path $\gamma:[0,1] \rightarrow M$ will be called $\omega$-positive if $\omega(\dot{\gamma}(t))>0$ for any $t \in[0,1]$. Calabi considered the following condition on $\omega$ which he calls transitivity:

(a) For any ordered pair of points $x$ and $y$ in $M$, which are not singular points of $\omega$, there exists an $\omega$-positive path from $x$ to $y$.

Evidently, (a) implies:

(b) For any non-singular point $x \in M$ there exists a closed $\omega$-positive path $\gamma$ through $x$.

We shall see that, in fact, (a) is equivalent to (b).

The main result of $[\mathrm{C}]$ can be reformulated as follows.

Theorem ([C]). A closed Morse 1-form $\omega$ on $M$ is harmonic with respect to some Riemannian metric if and only if condition (b) holds.

Remark. It is pointed out in $[\mathrm{C}]$ that (a) precludes the existence of critical points of index 0 or $n=\operatorname{dim} M$ and that non-singular forms satisfy (a).

\section{Singular FOLIATION OF A MORSE FORM}

4. Let again $\omega$ denote a closed 1-form with only Morse type singularities.

For any simply-connected open set $U \subset M$ we have $\left.\omega\right|_{U}=d f_{U}$ for some Morse function $f_{U}: U \rightarrow \mathbb{R}$ determined up to a constant. We will consider the foliation in $U$ determined by the level sets of $f_{U}$. Choosing a covering $\{U\}$ of $M$ we see that all these foliations match together to form a foliation $\mathcal{F}$ of $M$. We will call it the foliation determined by the form $\omega$.

Note this foliation has finitely many singular points which are the critical points of $\omega$. Locally the structure of the singular foliation $\mathcal{F}$ around a critical point of index $d$ has the form

$$
-x_{1}^{2}-\cdots-x_{d}^{2}+x_{d+1}^{2} \cdots+x_{n}^{2}=c
$$


A leaf of $\mathcal{F}$ is, by definition, any maximal subset $\mathcal{L}$ of $M$ such that for any two points $x, y$ in $\mathcal{L}$ there exists a smooth path $\gamma:[0,1] \rightarrow M$ which connects $x$ with $y$ and such that $\omega(\dot{\gamma}(t))=0$ for all $t$. There are finitely many leaves containing the singular points; we will call those leaves singular.

Note that the non-singular leaves, i.e. those containing none of the critical points of $\omega$, are smooth while the singular leaves are smooth except at each of the critical points which has a neighborhood homeomorphic to a cone over $S^{d-1} \times S^{n-d-1}$, where $d$ is the index of the critical point. Each nonsingular leaf $\mathcal{L}$ has a natural topology (which will be called the leaf topology) and smooth manifold structure such that the inclusion $\mathcal{L} \rightarrow M$ is an immersion; similarly for the singular leaves. We will be particularly interested in those leaves containing critical points of index 1 or $n-1$. In these cases removing a critical point locally disconnects the leaf and we will find it useful to define a singular leaf component to be the closure (in the leaf topology) of a connected component of $\mathcal{L}-\Sigma(\mathcal{L})$, where $\Sigma(\mathcal{L})$ denotes the set of all singular points of the leaf $\mathcal{L}$.

\section{CAlabi GRAPHS}

5. We now make some preliminary constructions to set up the proof of Theorem 1. In the next section 6 we will see that the Calabi properties of closed 1-forms can be interpreted by analogous properties of oriented graphs.

Let $\Gamma$ be an oriented connected finite graph. Consider the following two properties.

(a') If $x, y \in \Gamma$ (it suffices to consider only vertices) then there is a path from $x$ to $y$ that traverses edges of $\Gamma$ only in the positive direction.

(b') For any point $x \in \Gamma$, there exists a closed path through $x$ that traverses edges of $\Gamma$ only in the positive direction.

Clearly (a') implies (b').

Lemma 1. If $\Gamma$ satisfies ( $\left.b^{\prime}\right)$, then it satisfies $\left(a^{\prime}\right)$.

Proof. Condition (b') tells us that $\Gamma$ is the union of closed edge-paths which can be traversed in a positive direction with respect to the orientation. Since $\Gamma$ is connected, then, for any two points $x, y \in \Gamma$, there is a sequence of points $x=x_{1}, x_{2}, \cdots, x_{k}=y$ such that $x_{i}$ and $x_{i+1}$ are on a common closed edge path $C_{i}$. Clearly $x_{i}$ and $x_{i+1}$ can be joined by a positive path on $C_{i}$. Putting these together gives the desired path from $x$ to $y$.

Definition. We shall say that $\Gamma$ is a Calabi graph if it satisfies $\left(a^{\prime}\right) \sim\left(b^{\prime}\right)$.

In Figure 1 we show an example of a Calabi graph and a non-Calabi graph.

6. With any closed Morse 1-form $\omega$ on a closed manifold $M$, generating a singular foliation with all compact leaves, we shall associate an oriented graph $\Gamma_{\omega}$. With this goal in mind, we introduce an equivalence relation in $M$. We declare two points in $M$ to be equivalent if they lie on the same leaf of the foliation $\mathcal{F}_{\omega}$, generated by $\omega$. When all the leaves of $\mathcal{F}_{\omega}$ are compact, then the singular leaves are isolated (i.e. each singular leaf has a neighborhood, free of other singular leaves) and the quotient space $\Gamma_{\omega}:=M / \sim$ is a graph. Indeed, any non-singular leaf $\mathcal{L}$ has a collar, 


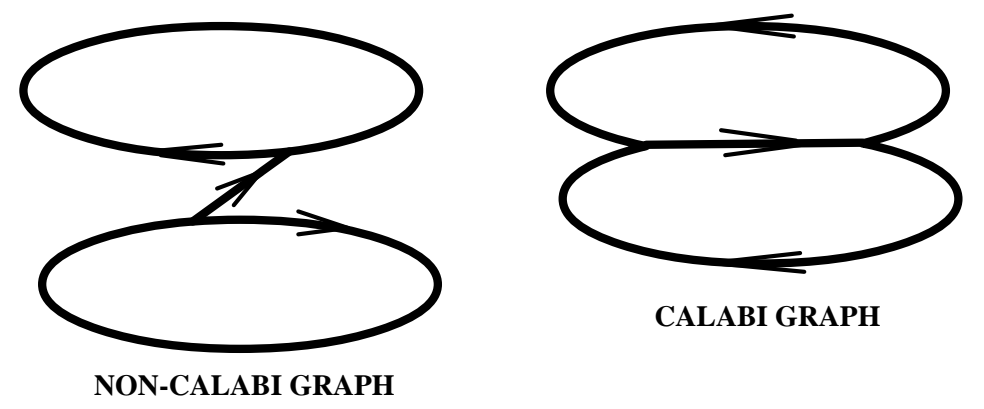

Figure 1

consisting of non-singular leaves. Hence, its neighborhood in the quotient space $\Gamma_{\omega}$ is an open interval. Suppose that $\mathcal{L}_{0}$ is a singular leaf corresponding to a point $v \in \Gamma_{\omega}$. The form $\omega$ is exact in some neighborhood of $\mathcal{L}_{0}$. Suppose $\omega=d f$ and $f^{-1}(a)=\mathcal{L}_{0}$. Then, for some $\epsilon,\left.f\right|_{f^{-1}(a, a+\epsilon)}$ and $\left.f\right|_{f^{-1}(a-\epsilon, a)}$ are fibrations and the fibers are non-singular leaves. Thus the complement of $\mathcal{L}_{0}$ in this neighborhood is a product of an open interval with a finite number of compact manifolds which define a finite number of edges of $\Gamma_{\omega}$ with $v$ viewed as a vertex.

Suppose $x_{i} \in \mathcal{L}_{0}$ is a critical point of index $s_{i}$. If $1<s_{i}<n-1$, then it follows from the local structure of a non-degenerate critical point that the intersection of each leaf with a small neighborhood of $x_{i}$ is connected. We conclude that if all the indices $s_{i}$ for a given singular leaf $\mathcal{L}_{0}$ satisfy $1<s_{i}<n-1$, then the leaves on both sides of $\mathcal{L}_{0}$ are connected and so $v$ is just an interior point of an edge of $\Gamma_{\omega}$. If $s_{i}=1$ or $n-1$ and $n>2$, then locally we have two non-singular leaves coalescing into the singular leaf from one side and one emerging on the other side (see Figure 2). (Of course it is possible that the what looks like two leaves locally may actually be part of the same leaf globally.)
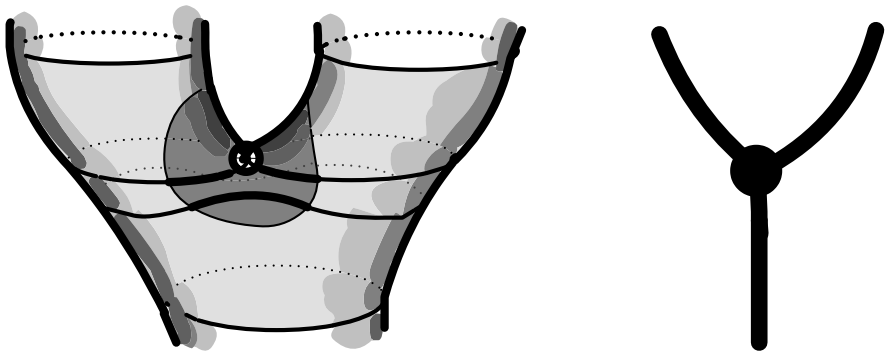

Figure 2

Thus when one or more of the indices $s_{i}$ equal 1 or $n-1$, then $v$ may be a true vertex of $\Gamma_{\omega}$. If $s_{i}=0$ or $n$, then we have non-singular fibers on only one side and $v$ will again be a true vertex.

In Figure 3 we show two examples of Morse 1-forms with all compact leaves whose associated graphs are those in Figure 1. The forms are the pullbacks of the canonical 1-form $d \theta$ on the circle $S$ via the obvious projection maps onto $S$.

The following lemma is obvious from the preceding discussion. 


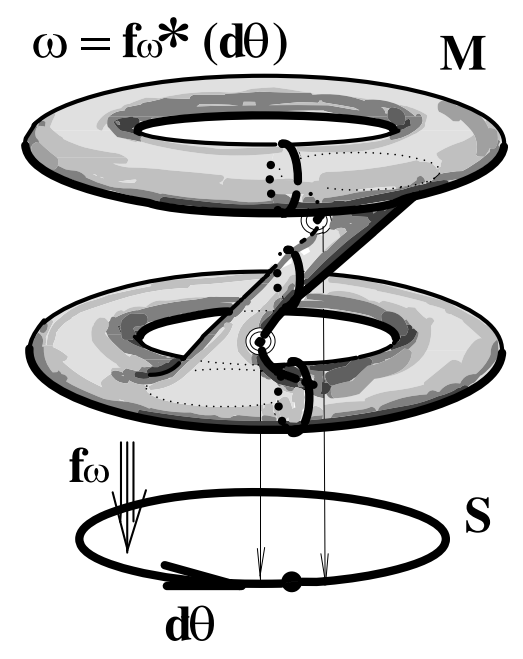

This map $f \omega$ into the circle is not intrinsically harmonic

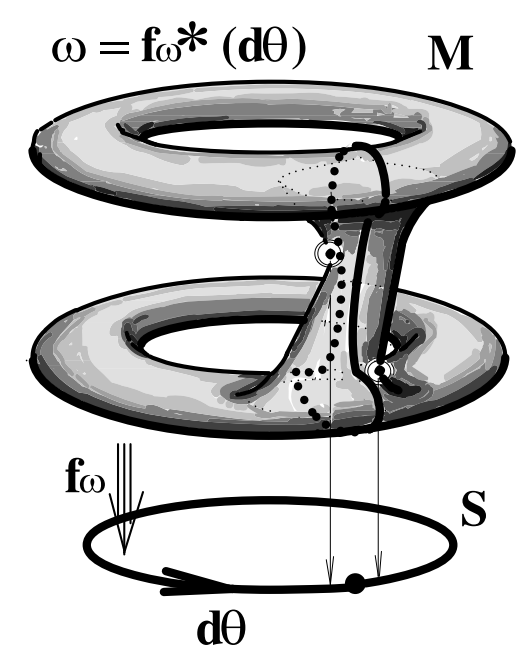

This map $f \omega$ into the circle is intrinsically harmonic

Figure 3

Lemma 2. $(M, \omega)$ (with all leaves compact) satisfies properties (a) and (b) of section 3 iff the associated oriented graph $\Gamma_{\omega}$ satisfies properties ( $\left.a^{\prime}\right)$ and ( $\left.b^{\prime}\right)$ of section 5 , respectively.

\section{ProOF OF THEOREM 1}

7. First, we remark that we may assume that $n=\operatorname{dim} M>2$ without loss of generality. In fact, if $n=2$, then from the Euler-Poincare Theorem it follows that any 1-form which has no maxima and minima, has precisely $-\chi(M)$ critical points of index 1 . Thus we will assume in the sequel that $n>2$. In fact, with some extra care, all our arguments will work in dimension 2. Our figures are all in dimension 2 so the reader should interpret them accordingly.

Multiplying the form $\omega$ by a scalar, we may assume that it is the differential of a (multivalued) Morse function $f: M \rightarrow S$, where $S$ is an oriented circle. More precisely, this means that $\omega=f^{*}(d \phi)$, where $d \phi$ is the standard angular form on the circle.

We may also assume, after a perturbation, that all the critical values of $f$ are distinct. Then, since there are no critical points of index 0 or $n$, every vertex of the graph $\Gamma_{\omega}$ (cf. section 6 ) is trivalent.

Consider the map $\psi_{f}: \Gamma_{\omega} \rightarrow S$ induced by $f$. Define the complexity of $\omega$ to be the smallest cardinality of any $\psi_{f}^{-1}(a)$, where $a$ ranges over the regular values of $f$. For example in Figure 3 the complexities of the two graphs are 2 and 1, respectively.

We will show that either:

(1) $\Gamma_{\omega}$ has no vertices, or

(2) there exists another closed 1-form $\omega^{\prime}$ with strictly smaller complexity such that $\omega$ and $\omega^{\prime}$ are contiguous. Recall, that this means, that $\omega$ and $\omega^{\prime}$ have the same numbers of critical points of all indices and belong to the same cohomology class. 
Thus, using induction, we will eventually reach case (1) i.e. we will find a closed 1 -form $\tilde{\omega}$ contiguous to $\omega$ and such that $\Gamma_{\tilde{\omega}}$ is a circle and $\psi_{f}$ is a covering map. In this case it is clear that $\Gamma_{\tilde{\omega}}$ is a Calabi graph and we are done.

Suppose that $\Gamma_{\omega}$ has at least one vertex. Choose a regular value $a$ such that the cardinality of $\psi^{-1}(a)$ equals the complexity of $\omega$. Cut open $M$ along $M_{a}=f^{-1}(a)$ to obtain a manifold $\bar{M}$. The map $f$ induces a Morse function $\bar{f}: \bar{M} \rightarrow[0,2 \pi]$ such that the two identical boundary components $M_{a}^{+}$and $M_{a}^{-}$map to 0 and $2 \pi$ respectively. The leaves of the foliation defined by $\bar{f}$ are the same as those of $\omega$ and the graph $\Gamma_{\bar{f}}$ it defines (cf. section 6 ) can be obtained from $\Gamma_{\omega}$ by cutting some of the edges. We now change the function $\bar{f}$, using the Morse-Smale reindexing technique (see $[\mathrm{M}]$ ), to obtain a self-indexing Morse function $\bar{g}: \bar{M} \rightarrow[0,2 \pi]$ with the same number of critical points of each index as $\bar{f}$ and such that $\bar{g}$ agrees with $\bar{f}$ in a neighborhood of $\partial \bar{M}$. The self-indexing property means that for any two critical points $x, y$, index $(x)>\operatorname{index}(y)$ implies $\bar{g}(x)>\bar{g}(y)$. We may also assume that all the critical values of $\bar{g}$ are distinct. If we reglue $M_{a}^{+}$and $M_{a}^{-}$, the function $\bar{g}$ induces a new Morse 1 -form $\omega^{\prime}$ on $M$ which is contiguous to $\omega$. See Figure 4.

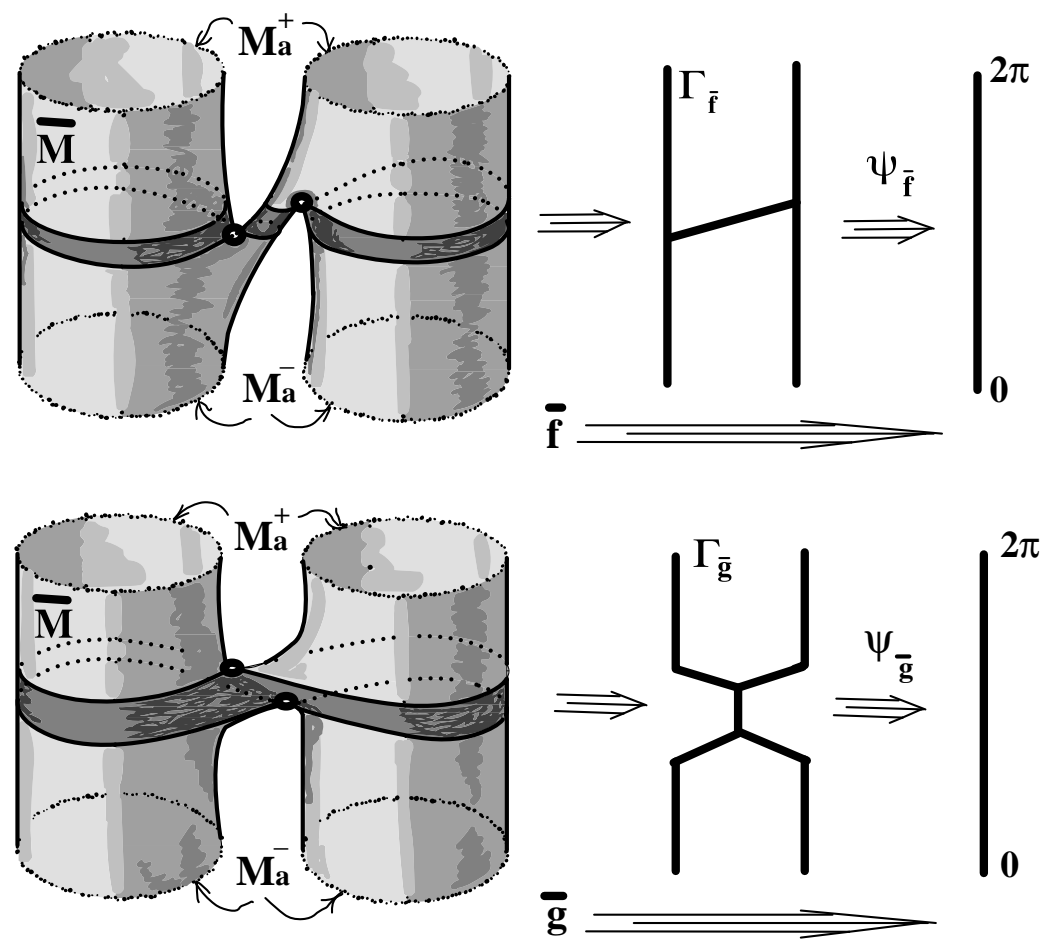

Figure 4

Now consider the associated oriented trivalent graphs $\Gamma_{\omega^{\prime}}$ and $\Gamma_{\bar{g}}$. The vertices of $\Gamma_{\bar{g}}$ are of two types: those with two ingoing and one outgoing edge (they necessarily belong to singular leaves containing critical points of index 1) and those with one ingoing and two outgoing edges (they necessarily belong to singular leaves containing critical points of index $n-1$ ). The self-indexing property implies (since we assume that $n>2$ and so $1<n-1$ that the induced map $\psi_{\bar{g}}: \Gamma_{\bar{g}} \rightarrow[0,2 \pi]$ has the property that there is some $b \in(0,2 \pi)$ such that $\psi_{\bar{g}}$ maps each vertex of the first type into 
$(0, b)$ and each vertex of the second type into $(b, 2 \pi)$. As a consequence, if there are any vertices at all, then the cardinality of $\psi_{\bar{g}}^{-1}(b)$ is strictly smaller than that of $\psi^{-1}(a)$. But this means that the complexity of $\omega^{\prime}$ is smaller than the complexity of $\omega$.

For example, applying this modification to the non-Calabi example in Figure 3 and its graph in Figure 1 will produce the form given by the other example in Figure 3 .

This completes the proof.

\section{Proof of Theorem 2}

8. Suppose the Morse 1-form $\omega$ has all non-compact leaves. We will show that it satisfies the Calabi condition (b) of section 3. Let $x \in M$. If $x$ belongs to a non-singular leaf, we will denote it by $\mathcal{L}_{x}$; if $x$ belongs to a singular leaf, then we will denote by $\mathcal{L}_{x}$ the singular leaf component (cf. section 4) containing $x$.

By statement (2) of Proposition 1 (cf. below in section 9), since $\omega$ is assumed to be generic, we know that $\mathcal{L}_{x}$ is non-compact.

First of all we point out that if any non-singular point $x^{\prime}$ of $\mathcal{L}_{x}$ satisfies Calabi condition (b), i.e. there is a $\omega$-positive closed path $\gamma$ passing through $x^{\prime}$, then any other non-singular point $x^{\prime \prime}$ in $\mathcal{L}_{x}$ satisfies Calabi condition (b) as well. To show this we connect $x^{\prime \prime}$ to $x^{\prime}$ by a path in $\mathcal{L}_{x}$. Now, $\gamma$ crosses $\mathcal{L}_{x}$ transversely at $x^{\prime}$ and so we can pull $\gamma$, as it passes through $x^{\prime}$, along $\gamma$, so that it now passes through $x^{\prime \prime}$. It is clear that we may keep $\gamma$ positive during this deformation. Thus, it suffices to find an $\omega$-positive closed path intersecting $\mathcal{L}_{x}$ anywhere.

Since $\mathcal{L}_{x}$ is not compact there exists a limit point $y$ of $\mathcal{L}_{x}$ not in $\mathcal{L}_{x}$. Since we have explicit models for the foliation near $y$, for the singular and non-singular case, we can see that all points on $\mathcal{L}_{y}$ close to $y$ are also limit points of $\mathcal{L}_{x}$. So we may assume that $y$ is not a critical point. If $U$ is a sufficiently small coordinate neigborhood of $y$, then the foliation on $U$ consists of parallel hyperplanes and so $\mathcal{L}_{x} \cap U$ must contain a sequence of these hyperplanes converging to the one containing $y$. Choose any two points $x^{\prime}, x^{\prime \prime}$ of $\mathcal{L}_{x} \cap U$ in different hyperplanes. We can connect these two points by a path $\rho:[-1,1] \rightarrow \mathcal{L}_{x}$ with $\rho(-1)=x^{\prime \prime}$ and $\rho(1)=x^{\prime}$. We can also obviously connect them by a positive path $\xi$ in $U$ (say, running from $x^{\prime}$ to $x^{\prime \prime}$ ). We now push $\rho$ slightly off $\mathcal{L}_{x}$ to make it into a positive path. To do so choose a vector field $X$ on $\mathcal{L}_{x}$ so that $\omega(X)$ is a positive constant. We can assume $X$ is tangent to $\xi$ at $x^{\prime}$ and $x^{\prime \prime}$. Define $\eta(t)=\gamma(t)+\epsilon t X(\gamma(t))$ for $\epsilon$ small enough, so that $\eta(-1)$ lies ahead of $\eta(1)$ on $\xi$. Now we can put $\eta$ together with the portion of $\xi$ that runs from $\eta(1)$ to $\eta(-1)$ to create a positive closed path which intersects $\mathcal{L}_{x}$ at $\eta(0)$. Of course, we need to round corners. See Figure 5.

9. Compact and non-compact leaves. It is clear from Theorems 1 and 2 that the general problem of deciding whether a given closed Morse 1-form is contiguous to a Calabi form may depend on the structure of the foliation defined by $\omega$. We will now explain how the manifold $M$ breaks up, in a nice way, into pieces made up (roughly) of all compact leaves or all non-compact leaves.

It is a theorem of S. Novikov, mentioned in [T], that when $\omega$ is non-singular (and $M$ is connected) then either all the leaves are compact, or all the leaves are non- 


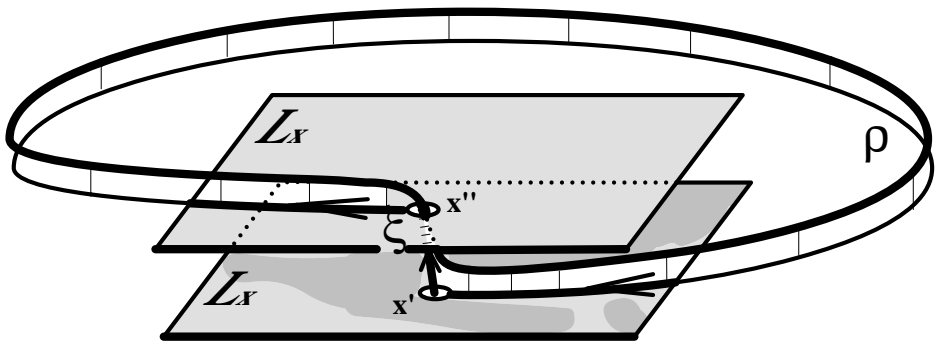

Figure 5

compact. This is certainly not true for general foliations (e.g. the Reeb foliation) but, in Proposition 1, we will generalize Novikov's theorem to foliations coming from a closed Morse 1-form, showing that the compact and non-compact leaves give a nice decomposition of $M$. The terms used in the statement were defined above in section 4 and in section 2 .

Proposition 1. If $\omega$ is a closed Morse 1-form on $M$, then $M$ is the union of two compact $n$-dimensional submanifolds $M_{c}$ and $M_{\infty}$, with a common (but generally singular) boundary, satisfying:

(1) Int $M_{c}$ is a union of all of the compact leaves plus some compact singular leaf components of non-compact leaves;

(2) Int $M_{\infty}$ is a union of all non-compact non-singular leaves, all non-compact singular leaf components and some compact singular leaf components of noncompact leaves.

(3) $\partial M_{c}=\partial M_{\infty}=M_{c} \cap M_{\infty}$ is a subvariety which is smooth except at a finite number of points, which are critical points of $\omega$, and is a union of some compact singular leaf components of non-compact singular leaves.

(4) If $\omega$ is generic, then $M_{c} \cap M_{\infty}$ contains all compact singular leaf components of non-compact leaves.

The theorem of Novikov follows from Proposition 1 since if there are no critical points, then by statement (3)

$$
\partial M_{c}=\partial M_{\infty}=\emptyset
$$

and so $M=M_{c}$ or $M=M_{\infty}$.

Proof. (1) Let $U$ be the union of all the compact leaves of the foliation determined by the form $\omega$. We show that $U$ is open. If $\mathcal{L}$ is a compact leaf, then it has a neighborhood $W$ with compact closure and containing no critical points other than those on $\mathcal{L}$, such that $\omega \mid \bar{V}=$ f for some smooth function on $\bar{V}$ with $f^{-1}(0)=C$. Then there exists $\epsilon>0$ such that $\overline{f^{-1}(-\epsilon, \epsilon)} \subseteq V$ and so $f^{-1}(a)$ is a compact leaf for $|a|<\epsilon$.

(2) Let $X$ be the union of all compact leaves and compact leaf components of non-compact leaves. Then $X$ is closed. Indeed if we consider the non-singular foliation $\mathcal{F}_{0}$ defined by $\omega$ on $M_{0}=M$-critical points of $\omega$, we can apply a theorem of Haefliger [H, p.386] to conclude that the union $X_{0}$ of all the closed leaves of $\mathcal{F}_{0}$ is a closed subset of $M_{0}$. But then $X$ is just the closure of $X_{0}$ in $M$. 
Let $M_{c}=\bar{U}$ and $V=M-\bar{U}$. From (2) we conclude that $Y=M_{c}-U$ is a union of some compact leaf components of non-compact leaves. $Y$ is a codimension one submanifold of $M$ except for singularities at the critical points of $\omega$ in $Y$. If $x$ is such a critical point of index $q$ then, for some neighborhood $W$ of $x,(W, Y \cap W) \cong$ cone over $\left(S^{n-1}, S^{q-1} \times S^{n-q-1}\right)$ if $1<q<n-1$, while if $q=1$ or $n-1$ then $(W, Y \cap W) \cong$ cone over $\left(S^{n-1}, S^{n-2}\right)$ or $(W, Y \cap W) \cong$ cone over $\left(S^{n-1}, S^{0} \times\right.$ $\left.S^{n-2}\right)$. In this last case we call $x$ special. It is clear that $M_{c}$ is a manifold with boundary $Y$ which is smooth except at the critical points and at those points we have a very explicit model of the singularity. At every point of $Y$, except the special points, $Y$ locally separates $M$ into two components exactly one of which must lie in $U$ and the other lies in $V$. At the special points $Y$ locally separates $M$ into three components if $n>2$ or four components if $n=2$. If $n>2$ then either one or two of the components lie in $U$. See Figure 6 .
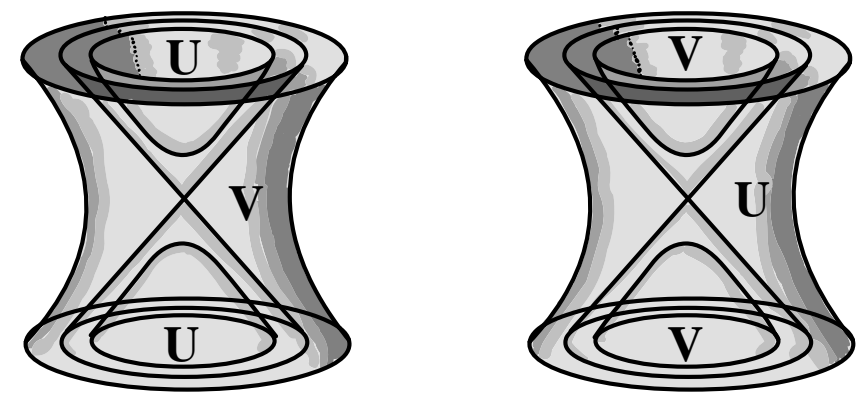

Figure 6

It remains to discuss the generic case. Suppose $C$ is a compact leaf component of a non-compact leaf $\mathcal{L}$. Then $C$ contains exactly one critical point $x$ which must be of index 1 or $n-1$. As above we have a neighborhood $W$ of $x$ so that $(W, \mathcal{L} \cap W) \cong$ cone over $\left(S^{n-1}, S^{0} \times S^{n-2}\right)$ where $C \cap W g$ ong one of the two cones over $S^{n-2}$. Now we can choose a regular neighborhood $R$ of $C$ so that $R=W$ near $x$ but $(R-W, C-W) \cong((C-W) \times[-1,1],(C-W) \times 0)$ since there are no other critical points on $C$. See Figure 7. It is clear that the leaves of the foliation defined by $\omega$ on one side of $C$ must lie entirely in $R$ and are homeomorphic to $C$. Since $C$ is compact this show $C \subseteq \bar{U}$.

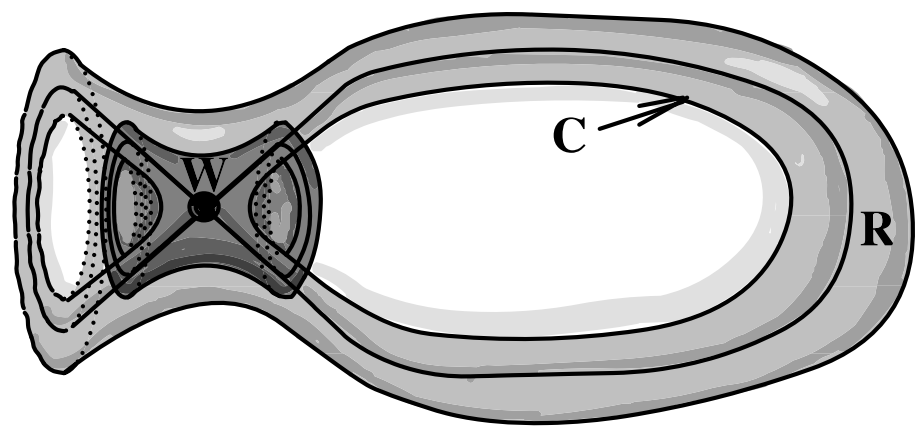


10. The next two propositions describe some relations between the cohomology class represented by a Morse 1-form and the presence of compact leaves in its foliation.

Proposition 2. A class $\xi \in H^{1}(M ; \mathbb{R})$ has a representative Morse 1-form with all compact leaves if and only if the homomorphism $\pi_{1}(M) \rightarrow \mathbb{R}$ determined by the cohomology class $\xi$ can be factorized $\pi_{1}(M) \rightarrow F \rightarrow \mathbb{R}$ through a free group $F$.

It will be convenient for us to refer to the cohomology classes satisfying the condition of Proposition 2 as split cohomology classes. A more geometric description of this condition is the following. The manifold $M$ can be cut into several pieces by disjoint codimension one submanifolds so that the restriction of the class $\xi$ to each piece is of rank one.

\section{Remarks.}

(i) As pointed out in Section 2, a rank one class has the property that every representative Morse 1-form has all compact leaves. In Section 12 we will give examples that suggest that only rank one classes have this stronger property.

(ii) It seems to be difficult to give a purely cohomological criterion for $\xi$ to be split, but a necessary condition is that there exist a finite subset $\left\{\xi_{i}\right\} \subseteq H^{1}(M ; \mathbb{Z})$ such that $\xi$ is a linear combination of the $\xi_{i}$ and all Massey products (see [D]) of the $\left\{\xi_{i}\right\}$ vanish. However this condition is not sufficient. An example can be obtained as follows. Choose a slice link $L$ of two components which is not a homology boundary link. According to [Hi, p.72] the link in Figure 8 is such an example. Define $M$ to be the result of 0 -surgery on $S^{3}$ along $L$. Then $H^{1}(M ; \mathbb{Z}) \cong \mathbb{Z}^{2}$ and all Massey products vanish. But there is no map $\pi_{1}(M) \rightarrow F, F$ a free group inducing an injection on $H^{1}$ and so, any class $\xi$ of rank two is not split.

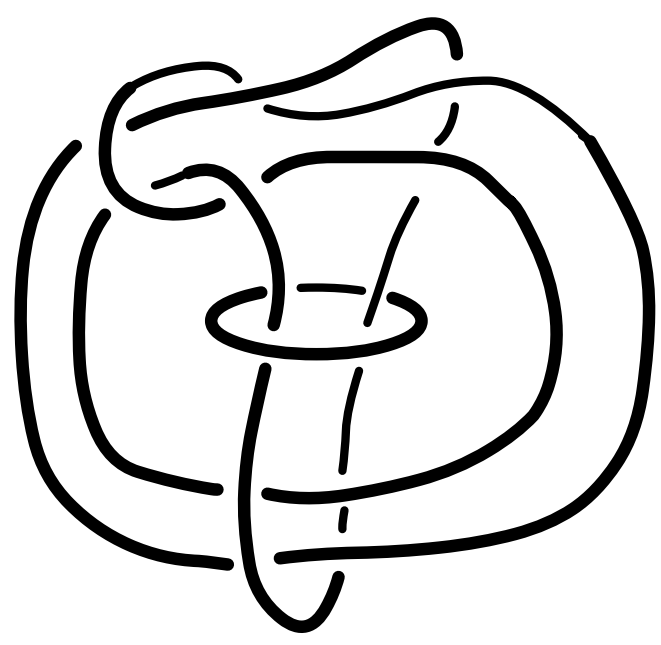

Figure 8

Proof of Proposition 2. Let $\omega$ be a Morse 1-form representing $\xi$ with all compact leaves and $\Gamma_{\omega}$ the associated oriented graph as defined in section 6 . Let $\phi: M \rightarrow \Gamma_{\omega}$ be the identification map. It is clear that $\xi \in \phi^{*} H^{1}\left(\Gamma_{\omega} ; \mathbb{R}\right)$ and half of Proposition 2 follows since any connected graph has a free fundamental group. 
Now suppose we are given $\xi$ and a map $f: M \rightarrow W$, where $W$ is a wedge of circles, such that $\xi=f^{*}\left(\xi^{\prime}\right)$ for some $\xi^{\prime} \in H^{1}(W ; \mathbb{R})$. Choose a point $a_{i}$ in each circle $S_{i}$ of $W$; we may assume that $a_{i}$ is a regular value of $f$. Then $M_{i}=f^{-1}\left(a_{i}\right)$ is a smooth compact submanifold of $M$. Let $\bar{M}$ be the result of cutting $M$ open along the $M_{i}$. Then $\partial \bar{M}$ consists of two copies $M_{i}^{+}$and $M_{i}^{-}$of each $M_{i}$. Obviously $\left.\xi\right|_{\bar{M}}=0$. Now assume each $S_{i}$ is oriented and set $p_{i}=\left\langle\xi^{\prime},\left[S_{i}\right]\right\rangle$, the period of the class $\xi^{\prime}$ on the circle $S_{i}$. We may assume that the orientations are such that a short path in $M$ which crosses $M_{i}$, hitting $M_{i}^{+}$before $M_{i}^{-}$, maps into $S_{i}$ with positive orientation. Now choose a Morse function $g: \bar{M} \rightarrow \mathbb{R}$ with no critical points on $\partial \bar{M}$, which is constant on each boundary component so that $g\left(M_{i}^{+}\right)-g\left(M_{i}^{-}\right)=p_{i}$. We may also assume that $\left.g\right|_{\partial \bar{M}}$ fits together smoothly to form a smooth 1-form $\omega$ on $M$. Clearly $\omega$ is Morse and closed, has zero periods in $\bar{M}$ and has period $p_{i}$ around any closed curve which crosses $M_{i}$ "positively" but no other $M_{j}$. Thus $\omega$ represents $\xi$. Furthermore, the leaves of the foliation defined by $\omega$ are components of $f^{-1}(a)$ for some $a \in \mathbb{R}$ and so they are compact.

11.

Proposition 3. If $\omega$ is a closed intrinsically harmonic Morse 1-form whose associated foliation has a compact leaf, then there exists some non-zero $\theta \in H^{1}(M ; \mathbb{Z})$ such that $\theta \cup[\omega]=0 \in H^{2}(M, \mathbb{R})$.

We will show in Section 12 that Proposition 3 is false if $\omega$ is not assumed to be intrinsically harmonic.

Proof. If $\omega$ has a compact leaf, then, by statement (1) of Proposition 1, there exist non-singular compact leaves. Suppose $\mathcal{L}$ is a non-singular compact leaf of $\omega$. Then $\mathcal{L}$ is a closed submanifold of $M$ and the normal bundle to $\mathcal{L}$ is trivialized by the form $\omega$. Also, $\mathcal{L}$ cannot bound in $M$ since this would contradict the Calabi condition.

We may find a cylinder $U=[-1 / 2,1 / 2] \times \mathcal{L}$ in $M$ fibred by the leaves of the foliation determined by $\omega$, such that the initially choosen leaf $\mathcal{L}$ is identified with $0 \times \mathcal{L}$. Now, let us construct a closed 1 -form $\alpha$ on $M$ such that:

(1) $\alpha$ vanishes outside the cylinder $U$;

(2) on the cylinder $U$ the form $\alpha$ is given by $\left.\alpha\right|_{U}=d(\phi \circ p)$ where $p: U \rightarrow$ $[-1 / 2,1 / 2]$ denotes the projection, and $\phi:[-1 / 2,1 / 2] \rightarrow[-1 / 2,1 / 2]$ is a smooth function satisfying:

$$
\begin{gathered}
\phi(t)=t \quad \text { for all }|t|<1 / 2-\epsilon \\
\phi(t)=1 / 2 \text { for all } t \in[1 / 2-\delta, 1 / 2] \\
\phi(t)=-1 / 2 \quad \text { for all } t \in[-1 / 2,-1 / 2+\delta]
\end{gathered}
$$

where $0<\delta<\epsilon$.

Thus we obtain that $\alpha$ represents a nonzero integral cohomology class $\theta \in$ $H^{1}(M, \mathbb{Z})$ and $\alpha \wedge \omega=0$. Therefore, on the level of cohomology, we get $\theta \cup[\omega]=0$ in $H^{2}(M, \mathbb{R})$.

Suppose, for example, that the pairing $H^{1}(M, \mathbb{R}) \times H^{1}(M, \mathbb{R}) \rightarrow H^{2}(M, \mathbb{R})$ is non-degenerate, i.e. for any $\xi \in H^{1}(M, \mathbb{R})$ there exists $\eta \in H^{1}(M, \mathbb{R})$ such that $\xi \cup \eta \neq 0$. Then it follows from Proposition 3 that, if a class $\xi \in H^{1}(M, \mathbb{R})$ is 
completely irrational, then any representative harmonic Morse 1-form has all noncompact leaves. (We say that a cohomology class $\xi$ is completely irrational if its rank is equal to the first Betti number of $M$.) This applies to the cases when $M$ is an orientable surface or $M=S^{1} \times \cdots \times S^{1}$.

\section{EXAMPLES}

12. We complement and illustrate Theorem 2 and Propositions 2 and 3 with some examples in dimension two. We first summarize the assertions of these results for a class $\alpha \in H^{1}(M ; \mathbb{R})$, where $M$ is a closed orientable surface, and a representative Morse 1-form $\omega_{\alpha}$.

(1) If rank $\alpha=1$, then any $\omega_{\alpha}$ has all compact leaves.

(2) $\alpha$ is split if and only if there exists some $\omega_{\alpha}$ with all compact leaves.

(3) If $\alpha$ is completely irrational and $\omega_{\alpha}$ is Calabi, then no leaves are compact.

(4) If $\omega_{\alpha}$ is generic and has no compact leaves, then $\omega_{\alpha}$ is Calabi.

We will construct the following four examples of $\alpha$ and $\omega_{\alpha}$ which will illustrate the sharpness of these results.

\section{Examples.}

(1) Rank $\alpha=$ one less than maximal and $\omega_{\alpha}$ Calabi with some compact leaves.

(2) Rank $\alpha>1, \alpha$ split and $\omega_{\alpha}$ Calabi with all leaves non-compact.

(3) a completely irrational and $\omega_{\alpha}$ non-Calabi with some compact leaves.

(4) $\omega_{\alpha}$ non-Calabi but all leaves non-compact (and so $\omega_{\alpha}$ is not generic).

We first describe three general connected sum operations which accept as input two closed 2-dimensional manifolds $M_{1}, M_{2}$ with closed Morse 1-forms $\omega_{1}, \omega_{2}$ and constructs a closed Morse 1-form $\omega$ on the connected sum $M_{1} \sharp M_{2}$ such that $\omega \mid M_{i}-$ $D_{i}=\omega_{i} \mid M_{i}-D_{i}$, where $D_{i}$ is any disk in $M_{i}$ containing no critical points of $\omega_{i}$ and within which the connected sum will be constructed.

Choose smooth functions $f_{i}: D_{i} \rightarrow \mathbb{R}$ such that $\omega_{i}=d f_{i}$. Suppose that we can identify each $D_{i}$ with an open rectangle $\left(a_{i}, b_{i}\right) \times\left(c_{i}, d_{i}\right)$ in $\mathbb{R}^{2}$ so that $f_{i}(x, y)=y$. Note that we can then move the rectangle by any translation in $\mathbb{R}^{2}$. Now we can perform a connected sum of $D_{1}$ and $D_{2}$ ambiently by connecting them with a straight tube in $\mathbb{R}^{3}$ in three different ways.

A. $f_{1}\left(D_{1}\right) \cap f_{2}\left(D_{2}\right)=J$ is non-empty, the connecting tube intersects $D_{i}$ inside $f_{i}^{-1}(J)$ and is approximately horizontal.

B. $f_{1}\left(D_{1}\right) \cap f_{2}\left(D_{2}\right)$ is empty,

C. $f_{1}\left(D_{1}\right) \cap f_{2}\left(D_{2}\right)$ is non-empty and the connecting tube has its two critical points at the same level.

See Figure 9.

We then define $f: D_{1} \sharp D_{2} \rightarrow \mathbb{R}$ to be the restriction of the height function. Clearly $d f$ blends with $\omega_{1}$ and $\omega_{2}$ to define a closed 1-form $\omega$ on $M_{1} \sharp M_{2}$. Note that in each of these constructions two new critical points $x, y$ of index 1 are introduced. In construction $\mathrm{A}, f(x)<f(y)$; in construction $\mathrm{B}, f(x)>f(y)$; in construction $\mathrm{C}$, $f(x)=f(y)$. 


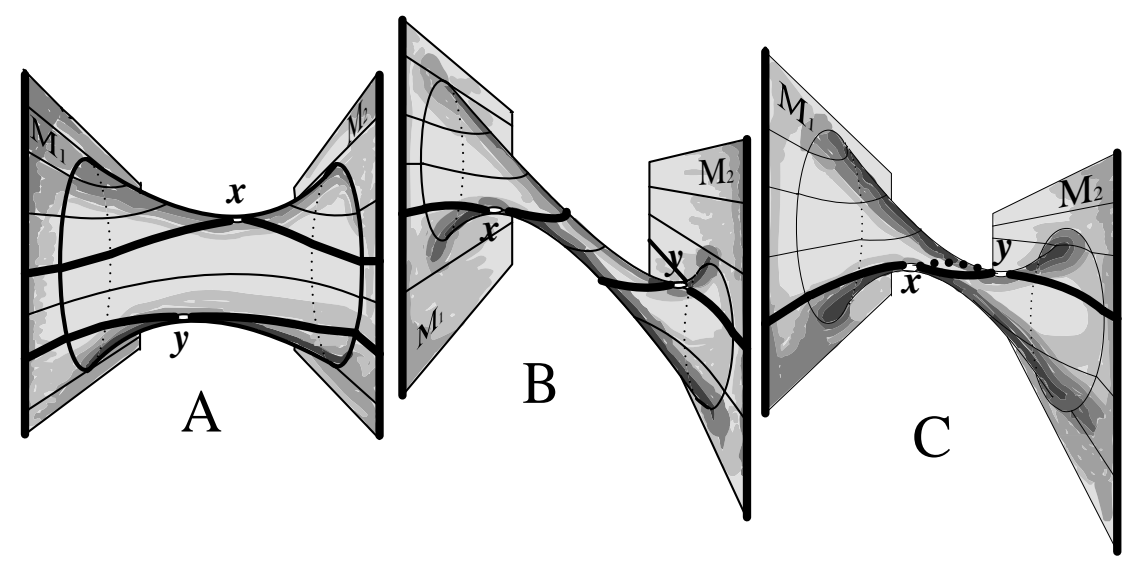

Figure 9

If we assume that the $\omega_{i}$ are Calabi then these constructions will have two important properties:

(1) Under construction A, $\omega$ is Calabi; under constructions B and C $\omega$ the consructed form is not Calabi.

(2) Under construction A, every leaf of $\omega$ intersects at least one leaf of $\omega_{1}$ and one leaf of $\omega_{2}$; under construction B there are new compact leaves produced; under construction $\mathrm{C}$ there is just one new compact singular leaf component produced.

We now use these general constructions to produce the four examples promised. In these examples we denote the torus by $T$ and $\theta, \phi$ will denote the usual angle coordinates on $T$.

Example 1. We construct a Calabi form with some compact leaves whose cohomology class has rank one less than the first Betti number. Let $M_{1}=T$ and $\omega_{1}=d \theta$ (or even $p d \theta+q d \phi$ for $p, q$ relatively prime integers). Let $M_{2}, \omega_{2}$ be arbitrary - for example $\omega_{2}$ might be a completely irrational Calabi form. Choose $D_{1} \subset M_{1}$ to be small enough so that many of the leaves of $\omega_{1}$ do not meet $D_{1}$. If we use construction $\mathrm{A}$, the result is a Calabi form of non-maximal rank with some compact leaves. See Figure 10.

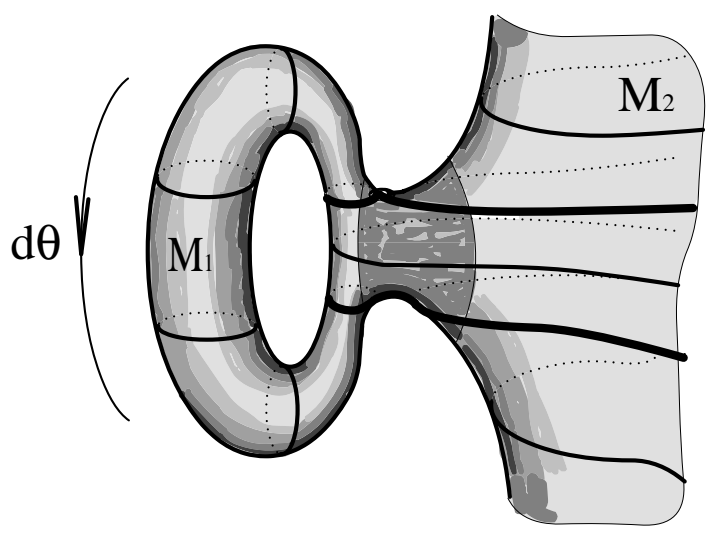

Figure 10 
Example 2. We construct a Calabi form with all non-compact leaves whose cohomology class is split but has rank $>1$. Let $M_{1}=M_{2}=T$ and $\omega_{1}=d \theta, \omega_{2}=$ $\lambda d \theta$, where $\lambda$ is irrational, and we use construction $\mathrm{A}$ to produce a Calabi form. If the disks $D_{1}$ and $D_{2}$ are small, then every leaf of $\omega_{1}$ or $\omega_{2}$ will hit them at most once and the leaves of $\omega$ will all be compact. If we choose the disks differently, though, so that they take the form of thin ribbons winding several times around $T$, then each leaf will hit the disk several times. The increments $\Delta f_{1}$ between different intersections of the same leaf of $\omega_{1}$ with $D_{1}$ will be integral multiples of $2 \pi$. The increments $\Delta f_{2}$ between different intersections of the same leaf of $\omega_{2}$ with $D_{2}$ will be integral multiples of $2 \pi / \lambda$. Since $\lambda$ is irrational, the attachments of leaves of $\omega_{1}$ with leaves of $\omega_{2}$ will produce non-compact leaves of $\omega$. See Figure 11.

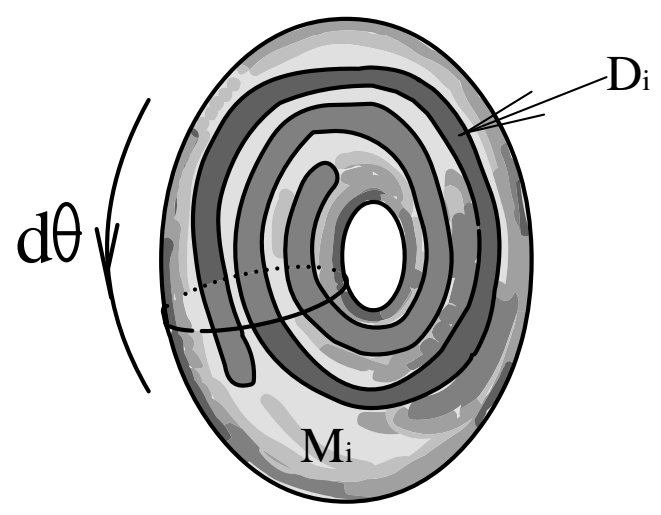

Figure 11

Example 3. We construct a non-Calabi form with some compact leaves whose cohomology class is completely irrational. Let $M_{i}$ and $\omega_{i}$ be arbitrary and use construction B. This will produce a non-Calabi Morse 1-form $\omega$ with some compact leaves. In this way choosing $\omega_{i}$ 's to be completely irrational with corresponding normalization so that $\omega$ will be completely irrational as well, we can realize any cohomology class on any surface except for a completely irrational class on a torus.

Example 4. We construct a non-Calabi non-generic form with all non-compact leaves. This is similar to example 3. Let $M_{1}=M_{2}=T$ and $\omega_{i}$ be any two forms of the form $a d \theta+b d \phi$, where $a / b$ is irrational. Then use construction $\mathrm{C}$ with small disks $D_{1}$ and $D_{2}$. This will produce a non-Calabi form with one compact singular leaf component and all non-compact leaves. See Figure 12.

13. Final Remarks. Theorems 1 and 2 still leave us with the problem of deciding, in general, whether closed Morse 1-forms are contiguous to harmonic forms. ${ }^{1}$ The next case to consider might be when the form $\omega$ has all compact leaves - the issue then reduces to a combinatorial question about the graph $\Gamma_{\omega}$. Settling the general compact leaf case and using Theorem 2 might then enable one to use Proposition 1 to deal with the general case.

\footnotetext{
${ }^{1}$ This has recently been settled in the affirmative by Ko Honda.
} 


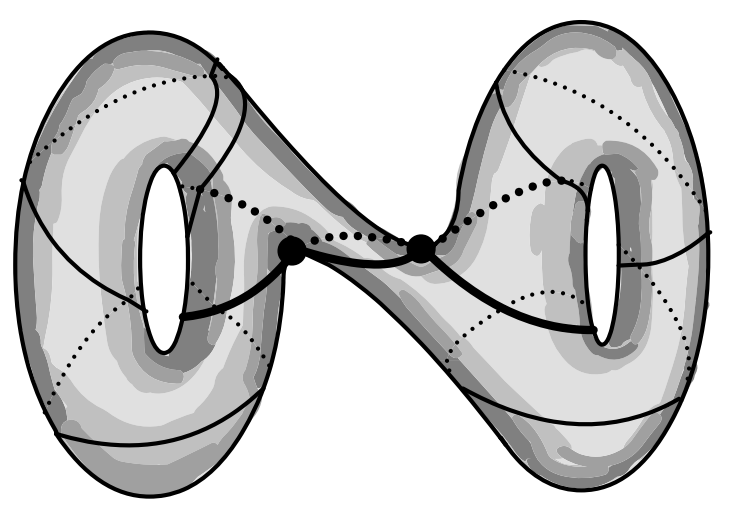

Figure 12

The results of Sections 10-11 and the examples of Section 12 leave open several interesting questions about the relation between the cohomology class of a closed Morse 1-form and the presence of compact leaves in its foliation.

(1) What conditions on a cohomology class will assure that it has a representative Morse form with no compact leaves? Example (2) suggests that rank $>1$ might be sufficient.

(2) What conditions on a cohomology class are satisfied if every representative Morse form has all leaves compact? Example (2) suggests that rank $=1$ is necessary.

(3) Is there some condition on a cohomology class which implies that every representative Morse form has all non-compact leaves? It is not hard to see that, on the torus, rank $>1$ will do. Example (1) shows that this is the only case for surfaces. In higher dimensions it is not hard to see that if a cohomology class $\alpha$ is completely irrational and unsplittable, in the sense that it is impossible to separate the manifold $M$ by a codimension one submanifold $V$ so that $\alpha \neq 0$ on each component of $M-V$, and if the pairing $H^{1}(M) \times H^{1}(M) \rightarrow H^{2}(M)$ is non-degenerate then $\alpha$ has the desired property.

\section{REFERENCES}

[C] E. Calabi, An intrinsic characterization of harmonic 1-forms, Global Analysis, Papers in Honor of K.Kodaira, (D.C.Spencer and S.Iyanaga, ed.), 1969, pp. 101 - 117.

[D] W. Dwyer, Homology, Massey products and maps between groups, Journal of Pure and Applied Algebra 6 (1975), 177-190.

[F] M. Farber, Exactness of the Novikov inequalities, Functional Anal. Appl. 19 (1985), 40-48.

[H] A. Haefliger, Variétés Feuilletées, Ann. Scuola Norm. Sup. Pisa 16 (1962), 367-397.

[Hi] J. Hillman, Alexander ideals of links, Springer Lecture Notes, vol. 895 (Springer-Verlag, ed.), 1981.

[LP] F. Lalonde and L. Polterovich, in preparation.

[M] J. Milnor, Lectures on the h-cobordism theorem, Princeton, New Jersey, 1965.

[N] S.P. Novikov, Multi-valued functions and functionals analogue of Morse theory, Soviet Math. Doklady 24 (1981), 222-226.

[N1] _ Hamiltonian formalism and multi-valued analogue of Morse theory, Russian Math Surveys 37:5 (1982), 1-56.

[P] A.V. Pazhitnov, Morse theory of closed 1-form, Lecture Notes in Math. 1474 (1991), Springer. 
[T] T. Tamura, Topology of foliations: an introduction, vol. 97, AMS Translations, 1992.

School of Mathematical Sciences, Tel-Aviv University, Ramat-Aviv 69978, Israel

Department of Mathematics, Clark University, Worcester, MA

Department of Mathematics, Brandeis University, Waltham, Ma

E-mail address: farber@math.tau.ac.il levine@math.binah.cc.brandeis.edu

gkatz@bbn.com 